\title{
Disc Instabilities and "Soft" X-Ray Transients
}

\author{
J.P. Lasota \\ UPR 176 du CNRS, DARC, Observatoire de Paris, Section de Meudon, \\ F-92195 Meudon Cedex, France
}

\begin{abstract}
I review several problems which arise when one tries to apply the standard dwarf nova instability model to outbursts of soft X-ray transients
\end{abstract}

\section{Introduction}

"Soft" X-ray transients (SXTs) are Low-Mass X-ray Binaries (LMXBs) which appear on the X-ray sky only for a couple of months. For years or decades they remain in a quiescent state in which the X-ray emission is just above, or more often below, the detectability threshold of present X-ray instruments. In LMXBs a neutron star or a black hole accretes matter lost by a Roche-lobe filling low-mass companion: a main sequence star (or a star already significantly evolved [King et al. 1996]) or a subgiant. In SXTs neutron stars are only weakly magnetized so that large scale magnetic fields play no role in the accretion which proceeds through a disc. The traditional name of "soft" X-ray transients may seem misleading because some of the transient luminosity is also observed in hard $\mathrm{X}$-rays, but most of the power is emitted in soft (or ultra-soft) X-rays, unlike the case of "Hard" X-ray transients which are High Mass X-ray Binaries (HMXBs) in which matter is accreted on to a strongly magnetized neutron star. On the other hand the frequently-used term "X-ray nova" is even more misleading since it suggests, incorrectly, that there is similarity between the X-ray transient event and nova eruptions.

The risetime of SXTs is 2 to 10 days and the outbursts last from 20 to 90 days. Many SXT light-curves can be described as "FREDs" (fast rise and exponential decay) but other, more symmetric forms have also been observed (Chen et al. 1996). Luminosities at outbursts are of the order of $10^{37}-10^{38} \mathrm{erg}$ $\mathrm{s}^{-1}$. The total energy emitted is typically $10^{43}-10^{44} \mathrm{ergs}$.

There is no doubt that the outburst is due to an increased accretion rate in the disc. Until recently two different mechanisms have been proposed to explain the cause of the transient events: a disc instability, analogous to the one proposed as the cause of dwarf nova (DN) outbursts, and a mass transfer instability in the secondary star that was supposed to be triggered by X-ray illumination. As was shown by Gontikakis \& Hameury (1993) (see also Lasota 1996a) the mass transfer instability model cannot account for the observed properties of SXTs, so that it should no longer be quoted as a possible model for SXT outbursts.

It is clear however that the DN disc instability model (DIM) cannot be applied to SXTs without modifications (Lasota 1996a,b; Cannizzo et al 1996; 
Mineshige 1996; Lasota et al. 1996; van Paradijs 1996). One should also stress that while X-ray illumination of the secondary cannot trigger the outburst, irradiation of the secondary and of the disc by $\mathrm{X}$-rays during the outburst may play an important role in the SXT phenomenon (Chen et al. 1994; Augusteijn et al 1994).

In this article I review possible modifications of the DIM that would allow one to apply it successfully to SXT outbursts. In particular I discuss the model in which the inner regions of the accretion flow are advection dominated (Narayan et al. 1996) and the role played in the model by X-ray illumination of the disc (van Paradijs 1996).

\section{The 'standard', dwarf-nova disc instability model}

The cause of the disc instability which operates in DNs is due to the partial ionization of hydrogen at $\sim 10^{4} \mathrm{~K}$. The resulting abrupt change in opacities between 6000 and $10000 \mathrm{~K}$ makes the disc (locally) thermally and viscously unstable. For accretion rates that correspond to this range of temperatures a stable equilibrium is impossible and one expects a limit-cycle behaviour. To obtain a global instability that would correspond to the observed amplitudes and durations of DN outbursts one has to assume that the viscosity $\alpha$ parameter depends on the local disc properties in such a way that it is 4 to 10 times larger in outburst than in quiescence. In the 'standard' disc instability model one assumes that the mass transfer from the secondary is constant during the outburst cycle.

The thermal instability model, however, cannot by itself account for the so called 'super-outbursts' observed in SU UMa systems. In this case an additional mechanism is needed to account for the higher amplitude and longer duration of the outbursts. Osaki (see this volume) has proposed a thermal-tidal disc instability model in which tidal forces increase the effective viscosity when the disc's outer radius is larger that a certain critical value. This model can work only for mass ratios (secondary/primary) smaller than $\$ 0.25$ and cannot therefore explain the high amplitude and very long outburst observed in U Gem (we do not call this unusual outburst a 'superoutburst', since no 'superhump' has been observed [and was not supposed to be observed; see Osaki in this volume], but its light-curve looks pretty much like a usual 'superoutburst').

In the case of systems in which only superoutbursts are observed (unlike the usual SU UMa stars, where superoutbursts are separated by cycles of 'normal' outbursts) the standard and the thermal-tidal disc instability models can be applied more or less successfully only if an additional assumption is made: the value of $\alpha$ in quiescence must be very small: $\sim 10^{-4}-10^{-5}$ (Smak 1993) instead of $\sim 10^{-2}$ in most of the DN systems. The physical reason for such low $\alpha$ values remains however unexplained.

The standard DIM also cannot account for the properties of many quiescent DN systems. According to DIM, in quiescence, the whole accretion disc has to sit on the cold branch of stable disc equilibria. This implies that the surface density $\Sigma$ of the cold disc must be lower than the value $\Sigma_{\max }$ corresponding to the maximum allowed effective temperature of a cold stable disc. The maximum surface density of the cold disc is approximated by

$$
\Sigma_{\max } \approx 672.8 \alpha_{0.01}^{-0.80} r_{10}^{1.10} m_{1}^{-0.37}
$$


the corresponding critical accretion rate, above which the disc is unstable, can be approximated as

$$
\dot{M}_{\text {crit,C }} \approx 4.7 \times 10^{15} r_{10}^{2.65} m_{1}^{-0.88} \mathrm{~g} \mathrm{~s}^{-1}
$$

(Hameury et al. 1996), where $\alpha=0.01 \alpha_{0.01}, m_{1}$ is the white dwarf mass in solar units and $r=10^{10} r_{10} \mathrm{~cm}$ is the radius in the disc.

In quiescence the local value of the accretion rate has to be everywhere smaller than $\dot{M}_{\text {crit,C }}(r)$, and the quiescent DN disc is not in equilibrium since $\dot{M} \neq$ constant.

Eq. (2) implies that for $m_{1}=1$ and a white dwarf radius $r_{10}=.05$ the accretion rate at the inner disc edge should be less than $\sim 6 \times 10^{12} \mathrm{~g} \mathrm{~s}^{-1}$. X-ray observations of quiescent $\mathrm{DNs}$, however imply values of the accretion rate on to the white dwarf of $\sim 10^{14}-10^{15} \mathrm{~g} \mathrm{~s}^{-1}$ (Erakleous et al. 1991; Mukai \& Shiokawa 1993), if the X-rays are emitted by the accretion flow, as seems indeed to be the case, since the secondaries are unlikely to emit the observed luminosity. As will be discussed below a similar problem arises for SXTs (Lasota 1996a,b; Cannizzo et al. 1995).

The standard DIM cannot therefore account for the properties of the inner accretion flow: according to the observations, the cold quiescent accretion disc cannot extend down to the surface of the white dwarf. A boundary layer emission is of no help since the required accretion rate on to the central object would be too high to satisfy the requirement of the DIM.

Since $\dot{M}_{\text {crit,C }}$ increases with radius while the accretion luminosity decreases, the difficulty can be solved by increasing the inner disc radius. Two ways of achieving this have been proposed. One (Livio \& Pringle 1992) is to assume that the white dwarf possesses a weak magnetic field which is strong enough to disrupt the inner disc in quiescence. In the other, at low accretion rates (low densities) the inner disc will evaporate, forming an extended coronal flow with a low radiative efficiency. A similar idea in the case of SXTs was proposed by Narayan et al. (1996). This advection-dominated accretion flow model (ADAF) will be discussed below. Of course in the case of a black hole the magnetic field hypothesis cannot work.

It is also interesting to note that 'removing' the inner disc regions may solve the so-called "optical - UV delay problem" in modeling outbursts of some DNs. In such DNs one observes a substantial delay in the optical and UV outbursts: the optical event begins first, and the UV one follows only after several hours. In the 'standard' DIM model the heating front propagates rather quite quickly from the outer (optical emission) disc towards the inner (UV emitting) disc regions and models cannot account for the observed delay. In a disc in which the inner regions have either evaporated or been disrupted by a magnetic field, the heating front will be stopped at the disc's inner edge, at which UV emission is weak. The inner disc reaching to the white dwarf surface will be rebuilt in viscous time, thus reproducing the observed delay (see Livio and Pringle 1992).

As has been mentioned above, if WZ Sge type outbursts were to be explained by the DIM one would have to assume an $\alpha$ in quiescence much smaller than in other DN outbursts. As shown by Hameury et al. in these proceedings an alternative model may explain WZ Sge properties without assuming unusually low $\alpha$ values. Also in this model one requires a truncated inner disc (see also 


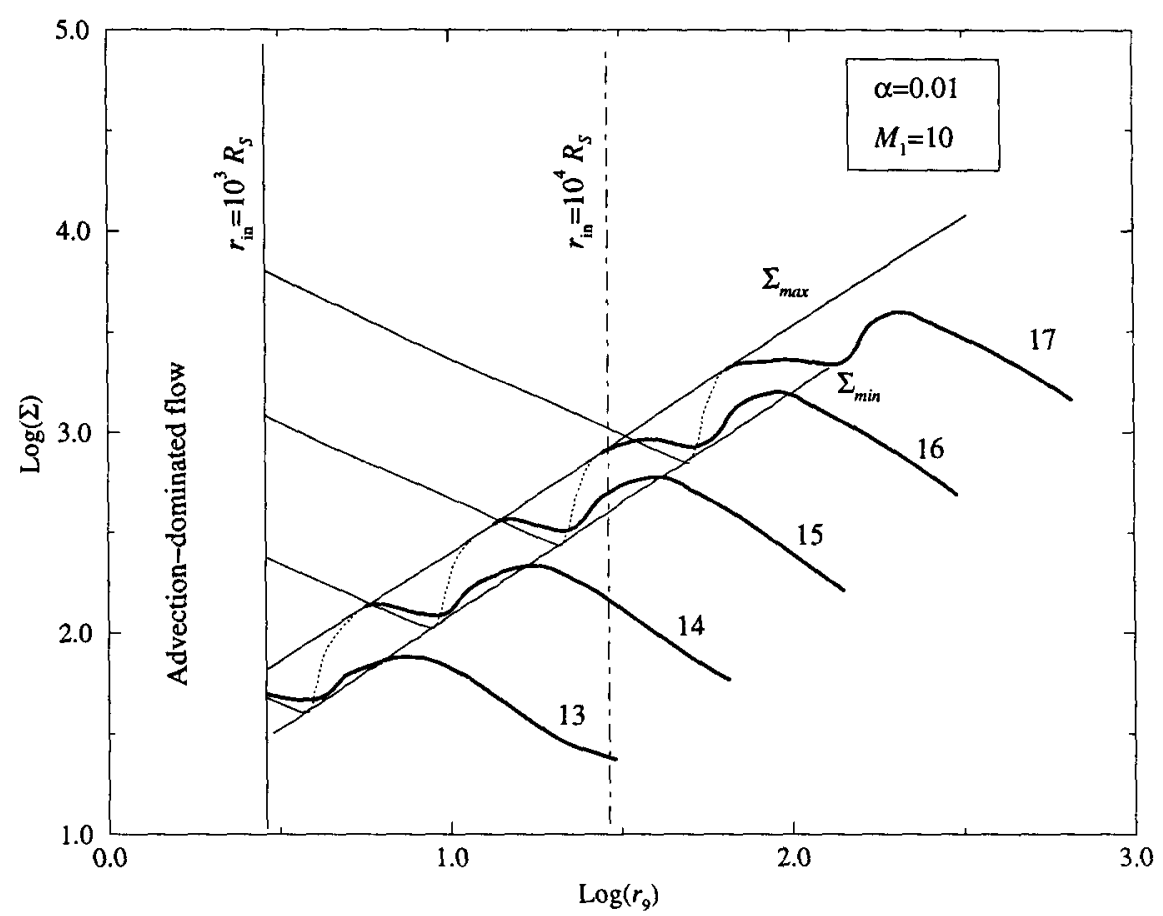

Figure 1. The surface density profiles of equilibrium accretion disc configurations around a black hole of $10 M_{\odot}$ with $\alpha=0.01$. Cold, stable equilibria are represented by solid lines, unstable by dotted lines. The hot stable solutions correspond to the straight lines terminating on the $\Sigma_{\min }$ line. The inner regions of thin discs could be truncated at $\sim 10^{3-4}$ Schwarzschild radii $\left(R_{s}\right)$.

Lasota et al. 1995). It is worth noting that WZ Sge systems are very similar to SXTs (Kuulkers et al. 1996; Lasota 1996a,b).

One can conclude therefore that in many cases the DIM has to be modified to be able to account for the properties of DN outbursts. The required modifications concern the inner disc regions. Similar problems are encountered when the DIM is applied to SXT outbursts.

\section{The disc instability model for SXTs}

\subsection{The stability criterion}

At effective temperatures $T_{\text {eff }}>T_{\text {crit }} \approx 6500 \mathrm{~K}$ an accretion disc is thermally stable (on the 'hot' branch). Since the effective temperature for a stationary 
accretion disc is given by

$$
T_{\text {eff }}=\left(\frac{3 G M \dot{M}}{8 \pi \sigma r^{3}}\right)^{1 / 4}
$$

where $\sigma$ is the Stefan-Boltzmann constant, it is enough for stability that $T_{\text {eff }}>$ $T_{\text {crit }}$ at the outer disc radius. On Fig. 1 the hot stable solutions are represented by straight lines terminating on the $\Sigma_{\min }$ line, where $\Sigma_{\min }$ corresponds to the minimum surface density of the hot solution. From Eq. (3) and the relation between the disc radius and the orbital period one can obtain an expression for the critical mass transfer rate above which, for a given orbital period, the disc is stable (Smak 1983; see also King et al. 1996):

$$
\dot{M}_{\text {crit,H }} \approx 1.8 \times 10^{17} P_{3}^{2} \mathrm{~g} \mathrm{~s}^{-1}
$$

where $P_{3}=P /(3 \mathrm{hr})$. In deriving Eq. (4) the relation

$$
r_{\mathrm{D}} \approx 3.0 \times 10^{10} \mathrm{~m}_{1}^{/ 3} P_{3}^{2 / 3} \mathrm{~cm}
$$

has been used, where $r_{D}$ is the outer disc radius.

Figure 1 illustrates the significance of the stability criterion given by Eq. (4). One can see, for example, that the straight line part of the equilibrium curve for $\dot{M}=10^{17}$ ends on the $\Sigma_{\min }$ line at a radius $\sim 6.5 \times 10^{10} \mathrm{~cm}$, which, according to Eq. (5), corresponds to $P \approx 3 \mathrm{~h}$, in agreement with Eq. (4).

The criterion $\dot{M}>\dot{M}_{\text {crit,H }}$ is a sufficient condition for disc stability (or its negation a necessary condition for instability). It is not a necessary condition for stability since a disc for which $\dot{M}<\dot{M}_{\text {crit,C }}\left(r_{\text {in }}\right)$ (see Eq. 2) is globally stable since it is everywhere on the 'cold' stable branch. If one assumes, as does the standard version of the DIM, that the disc extends down to the white dwarf surface, mass transfer rates are considered to be too low $\left(\dot{M} \lesssim 10^{12}-10^{13} \mathrm{~g}\right.$ $\mathrm{s}^{-1}$ ) to be of practical interest and $\dot{M}>\dot{M}_{\text {crit,DN }}$ is assumed to be a necessary and sufficient stability condition.

In the case of some dwarf novae (as discussed above) and for all black-hole SXTs (BHSXTs) the inner accretion disc has to be truncated (as we will show below) so the condition $\dot{M}>\dot{M}_{\text {crit }, \mathrm{H}}$ is not a necessary condition for stability even in practical applications. For WZ Sge Lasota et al. (1995) considered a model in which the inner disc is truncated at $r_{\mathrm{tr}} \approx 2.5 \times 10^{9} \mathrm{~cm}$ (for $m_{1}=0.4$ ) and thus marginally stable (see also Warner et al. 1996 and Hameury et al. in these proceedings). Narayan et al. (1996) proposed a quiescent SXT model in which the outer dwarf-nova type disc is truncated at $10^{3}-10^{4} r_{\mathrm{S}}$, where $r_{\mathrm{S}}=2 G M / c^{2} r$ is the Schwarzschild radius, so that this outer disc could be globally stable for mass-transfer rates $\dot{M} \sim 10^{14}-10^{16} \mathrm{~g} \mathrm{~s}^{-1}$ (see e.g. Lasota et al. 1996 and Narayan in these proceedings). These models will be discussed in the next section. Before that, in the next subsection, we will discuss how $\mathrm{X}$-ray illumination may affect the stability criterion.

\subsection{The effect of $\mathrm{X}$-ray illumination on the disc stability}

The stability criterion requiring mass transfer rates to be larger than the value given by Eq. (4) is derived from the requirement that the disc's effective temperature be higher than $\sim 6500 \mathrm{~K}$ everywhere. In the standard DIM it is assumed 


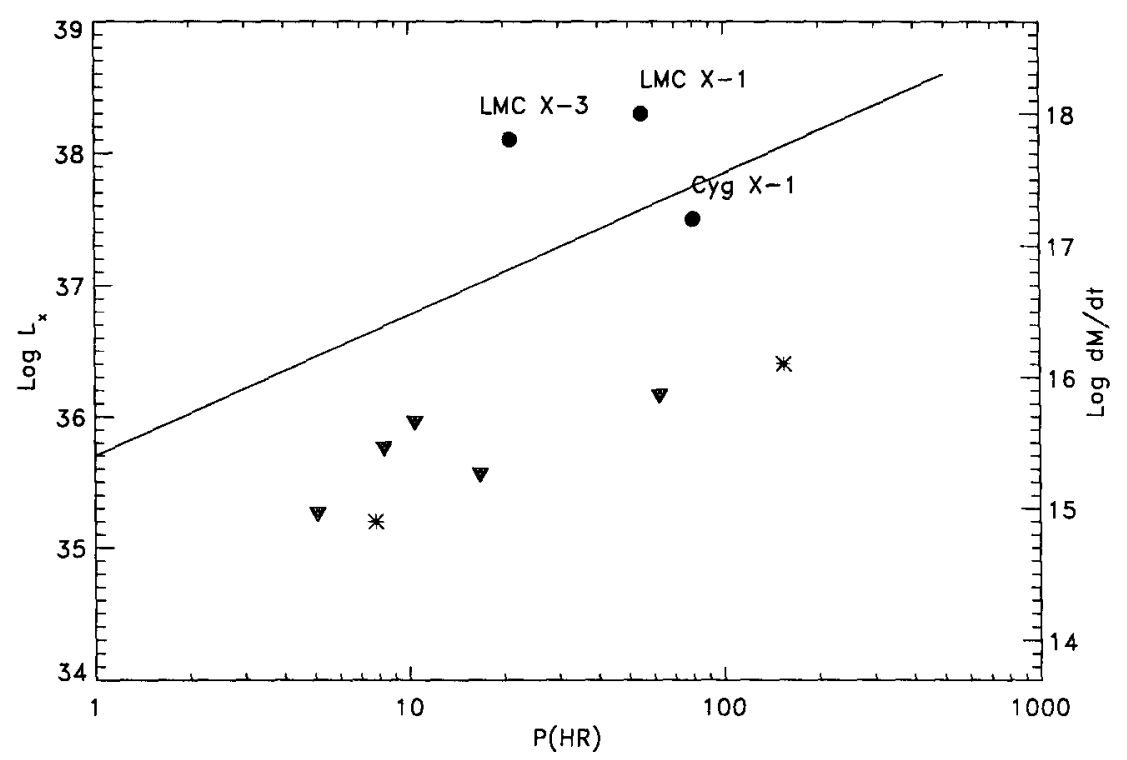

Figure 2. X-ray luminosity (and average mass transfer rate) as a function of orbital period for persistent and transient LMXB with black holes. The transients with known recurrence times (A0620-00 and V404 Cyg) have been indicated with an asterisk, the other transients with a triangle. The straight line indicates the separation between persistent and transient sources derived here for black holes of $10 \mathrm{M}_{\odot}$ (Eq. 6). The figure also includes (indicated by dots) the three persistent high-mass X-ray binaries Cyg X-1, LMC X-1 and LMC X-3, at the fiducial positions that they would have occupied if they had been LMXB with an equally large accretion disc, i.e., Roche lobe of the X-ray source; (from van Paradijs 1996).

that the disc temperature is determined by power released by viscosity (see Eq. (3)).

Recently van Paradijs (1996) pointed out that since accretion discs in LMXBs are likely to be strongly affected by X-ray illumination from the central source the heat released by the X-ray irradiation should stabilize the disc, so that LMXB accretion discs would be stable at accretion rates lower than those given by Eq. (4). By taking $\mathrm{X}$-ray heating into account, van Paradijs (1996) obtained a new critical mass transfer rate above which accretion discs in LMXB are globally stable. This critical rate can be written as (King et al. 1996):

$$
\dot{M}_{\text {crit,irr }} \approx 3.2 \times 10^{15} \mathrm{~m}_{1}^{2 / 3} \mathrm{P}_{3}^{4 / 3} \mathrm{~g} \mathrm{~s}^{-1}
$$

The new critical $\dot{M}$ is represented on Fig. 2. on which the parameters of black hole X-ray binaries are plotted. The new criterion seems to separate persistent 
sources from transient ones (or variable like Cyg X-1). The same is true for LMXBs containing neutron stars (van Paradijs 1996).

Like Smak's criterion for DNs, the van Paradijs criterion gives a sufficient condition for stability: it states that a stationary disc for which $\dot{M}>\dot{M}_{\text {crit,irr }}$ is globally stable. Unlike Smak's criterion, the van Paradijs condition is non-local: the X-ray illumination of the outer disc depends on the accretion rate on to the central object and on the shape of the disc, which must allow the outer disc "to see" the central X-ray source. A stationary disc is concave (in the range of parameters of interest) so the outer disc is exposed to the irradiation by the central source.

This is not the case of non-stationary quiescent discs according to the DIM. There, because of the convex disc shape, its outer regions are shielded from the central X-ray source (Cannizzo 1994). So, do transient sources obey the criterion $\dot{M}<\dot{M}_{\text {crit,irr }}$ ? This will depend on their history, how they have arrived at the present level of mass transfer rate. If the present mass transfer rate was achieved by going through a sequence of mass transfer rates higher than the present one, the van Paradijs criterion will be obeyed. If, however, the present state was achieved from lower mass transfer rates, i.e. through a sequence corresponding to globally unstable disc configurations, the mass transfer rates could be higher than the critical value given by Eq. (6) because the outer disc would never be exposed to the central X-ray source (Cannizzo 1994).

A quick look at Figure 2. shows, however, that (estimated) mass transfer rates for BHSXTs are in fact well below the limit given by Eq. (6). On the other hand, all persistent sources are above the van Paradijs limit and no SXT has a mass transfer rate superior to $\dot{M}_{\text {crit,irr, }}$ confirming that this critical value provides a sufficient condition for disc stability. This does not mean, however, as we will see below, that accretion discs in BHSXT are unstable.

\subsection{The standard DIM applied to SXTs}

The application of the standard DIM to SXT outbursts (Mineshige \& Wheeler 1989; Cannizzo et al. 1995) leads to the same difficulties as those encountered in the case of X-ray emitting quiescent dwarf novae (see section 2) but these difficulties are more acute. The DIM, in its standard version, requires the whole disc to be in the cold, stable equilibrium. Since the critical accretion rate (Eq. 2) scales as $r^{2.5}$ and a black hole (or neutron star) radius is about $10^{3}$ times smaller than a white dwarf radius, the quiescent accretion rates on to the central body required by the DIM are, in the case of SXTs, ridiculously small $\left(\$ 10^{6} \mathrm{~g} \mathrm{~s}^{-1}\right)$ and in contradiction with observations.

Indeed, several BSXTs have been observed by GINGA (Mineshige et al. 1992) and ROSAT (see Verbunt 1996), and two systems, A0620-00 and V404 Cyg, have been detected at levels corresponding to accretion rates (assuming an efficiency of 0.1 , see below) of at least $\sim 1.3 \times 10^{11} \mathrm{~g} \mathrm{~s}^{-1}$ for A0620-00 and $3 \times 10^{12} \mathrm{~g} \mathrm{~s}^{-1}$ for V404 Cyg (Mineshige et al. 1992; McClintock, et al. 1995; Verbunt 1996). These accretion rates are several orders of magnitude larger than the values of $\lesssim 10^{6} \mathrm{~g} \mathrm{~s}^{-1}$ required by the standard DIM.

We note that in the case of A0620-00, the prototypical BSXT, the optical and UV luminosities suggest a mass transfer rate of $\sim 6 \times 10^{15} \mathrm{~g} \mathrm{~s}^{-1}$ (McClintock et al. 1995). 
One could argue that since the observed $\mathrm{X}$-ray fluxes are low (in the case of A0620-00 only 39 counts were detected) there is no reason to assume that these $\mathrm{X}$-rays are emitted by the accretion flow. One can, however, exclude (Verbunt 1996) that X-ray emission from the companion (except for A0620-00 for which the evidence is marginal). Future observations will have to decide on the origin of the X-rays observed in quiescent SXTs (V404 Cyg is here the most promising system). However, as mentioned above (see Cannizzo et al. 1995), the problem of inconsistency between requirements of the DIM and observations is analogous to the one encountered for $\mathrm{DNs}$ so we would find it surprisising if the $\mathrm{X}$-rays were not emitted by matter accreting on to the central object.

\section{Advection dominated accretion flows in SXTs}

Let us forget for the moment about the requirements of the DIM and consider the implications of the X-ray and optical/UV observations for the structure of quiescent accretion flows in SXTs. If we assume that the accretion flow forms a Shakura-Sunyaev disc down to the last stable orbit around the black hole, the efficiency of accretion is (in the Newtonian approximation, which is sufficient for the order of magnitude arguments) $\eta \sim G M / r c^{2}$, i.e $\sim 0.1$ for a black hole. One can then deduce accretion rates from luminosities by using the relation $\dot{M}=\eta L / c^{2}$. In such a 'standard' framework the mass transfer rate in A0620-00 is $6 \times 10^{15} \mathrm{~g} \mathrm{~s}^{-1}$ whereas the accretion rate into the black hole is $\sim 3 \times 10^{11} \mathrm{~g}$ $\mathrm{s}^{-1}$ (McClintock et al. 1995). So there is a four orders of magnitudes difference between the rate in which matter is deposited at the outer disc edge and the rate at which it is lost in the black hole. The large differences between mass transfer rates and accretion rates on to the central accreting object are typical of quiescent, nonstationary discs of the DIM, which leads McClintock et al. (1995) to the conclusion that their observations of A0620-00 confirm the DIM. As was mentioned in the previous section however, the DIM requires a 10 orders of magnitude difference, so that in fact McClintock et al.'s (1995) observations refute the DIM for SXTs.

The difficulty in modelling quiescent SXT discs is due to the discrepancy between the 'observed' mass transfer rate and the accretion rate on to the central object deduced by assuming a radiative efficiency of 0.1 . This discrepancy cannot be explained in the framework of the DIM. There are other systems in the Universe from which such discrepancy is inferred: our Galactic Center, nuclei of giant elliptical galaxies and some weak active galactic nuclei. As this was first realized by Rees (1982), in such systems the efficiency for conversion of rest mass into luminosity may be rather low, i.e. much lower than 0.1 , if only a small fraction of the heat released by accretion can be radiated away during the infall of the material on to the black hole. In such advection-dominated accretion flows (ADAFs) the accretion rate on to the black hole is obviously much higher than the one deduced by using a 0.1 efficiency and in particular the accretion rate can be constant in the flow. Fabian \& Rees (1995) have suggested that accretion flows in the nuclei of giant elliptical galaxies are advection-dominated.

Recent work on optically thin ADAFs (see Narayan in these proceedings) provided reliable models that can be used to describe various systems. 
The two-temperature model of Narayan \& Yi (1995) has been applied to to the Galactic Center (Narayan et al. 1995), and the LINER NGC 4258 (Lasota et al. 1996) [in this case, there are, model-dependent, arguments against this object containing an ADAF - see articles by Maloney and by Begelman in these proceedings]. Narayan et al. (1996) proposed a model of quiescent SXTs in which an outer cold Keplerian disc has its inner edge at a large transition radius $\left(10^{3}-10^{4} r_{S}\right)$ at which the flow becomes very hot and advection dominated. Mineshige (1996) used such a framework to discuss the mechanism driving SXT outbursts.

In the Narayan et al. (1996) model the accretion rate is constant through the disc. The $\mathrm{X}$-rays are emitted by the ADAF while the UV/optical radiation originates in the outer cold disc. As was shown by Lasota et al. (1996), however, the UV/optical flux cannot be emitted by this part of the disc. This is a problem for all models. In the new version of this model presented at this meeting by Narayan, most of the observed radiation is emitted by the ADAF with a transition radius at about $6000 R_{S}$ for A0620-00 and $25000 R_{S}$ for V404 Cyg (Narayan et al. 1997) but the value of transition radius is not very well constrained by the models. The UV flux is due to the synchrotron emission of the ADAF. The outer disc is stationary.

If the outer disc is indeed stationary, what is the origin of the outbursts? Kuulkers et al. (1996) claim that the outburst mechanism must be the same both in WZ Sge systems and in SXTs. I agree with them, but disagree about the cause. As in the Hameury et al. model of WZ Sge, the outbursts of SXTs could be due to an enhanced mass transfer rate (see e.g. Lasota 1996b) which brings the marginally stable disc into an unstable regime. It is interesting to note that the positions of the BHSXTs in Fig. 2. correspond to marginally stable discs truncated at $r_{\mathrm{tr}} \approx 10^{3}-10^{4} r_{S}$, i.e. values required, by the Narayan et al. model. As can be seen from Fig. $1 r_{\mathrm{tr}} \approx 10^{4} r_{S}$ corresponds to a stable outer disc for $\dot{M} \gtrsim 10^{16} \mathrm{~g} \mathrm{~s}^{-1}$ and for $\dot{M} \gtrsim 10^{15} \mathrm{~g} \mathrm{~s}^{-1}$ the outer disc is stable for $r_{\mathrm{tr}} \gtrsim 3 \times 10^{3} r_{S}$.

Acknowledgments. I am gratful to John Cannizzo, Jean-Marie Hameury, Andrew King, Ramesh Narayan and Craig Wheeler for enlightening discussions on the subject of SXTs.

\section{References}

Augusteijn, T., Kuulkers, E., \& Shaham, J. 1993, A\&A, 279, L13

Cannizzo, J.K. 1993, in Accretion Disks in Compact Stellar Systems, ed, J.C. Wheeler, (Singapore: World Scientific), 6

Cannizzo, J.K., 1993, ApJ, 419, 318

Cannizzo, J.K., 1994, ApJ, 435, 389

Cannizzo, J.K., Chen, W., \& Livio, M., 1995, ApJ, 454, 880

Chen,W., Gehrels, N., \& Livio, M., 1993, ApJ, 408, L5

Chen,W., Shrader, C.R., \& Livio, M., 1996, ApJ, submitted

Eracleous, M., Halpern, J., \& Patterson, J. 1991, ApJ, 382, 290

Fabian, A.C.,\& Rees, M.J. 1995, MNRAS, 275, 641 
Gontikakis, C., \& Hameury, J.-M., 1993, A\&A, 271, 118

Hameury, J.-M., Huré, J.-M., Lasota, J.-P. 1996, A\&A, in preparation

King, A.R., Kolb, U., \& Burderi, L. 1996, ApJ, 464, 131

Kuulkers, E., Howell, S.B., \& van Paradijs, J. 1996, ApJ, 462, L87

Lasota, J.-P. 1996a, in IAU Symp. 165, Compact Stars in Binaries, ed. E.P.J. van den Heuvel et al. (Dordrecht: Kluwer), 43

Lasota, J.-P. 1996b, in IAU Coll. 158, Cataclysmic Variables and Related Objects, ed. J.H. Wood et al. (Dordrecht: Kluwer), in press

Lasota, J.-P., Hameury, J.-M. \& Huré, J.-M. 1995, A\&A, 302, L29

Lasota, J.-P., Narayan, R., \& Yi. I. 1996 A\&Ain press

Lasota, J.-P., Abramowicz, M.A., Chen, X.M., Krolik, J.H., Narayan, R., \& Yi., I. 1996, ApJ, 462, 142

Livio, M., \& Pringle, J.E. 1992, MNRAS, 259, 23P

McClintock, J.E., Horne, K., \& Remillard, R.A. 1995, ApJ, 442, 358

Mukai, K. \& Shiokawa, K. 1993, ApJ, 418, 863

Meyer, F. \& Meyer-Hofmeister, E., 1994, A\&A, 288, 175

Mineshige, S. 1995, PASJ, 48, 93

Mineshige, S. \& Wheeler, J.C. 1989, ApJ, 343, 241

Mineshige et al. 1992, PASJ, 44, 117

Narayan, R., \& Yi, I. 1995, ApJ, 452, 710

Narayan, R., Yi, I., \& Mahadevan, R. 1995, Nature, 362, 820

Narayan, R., McClintock, J.E., \& Yi, I. 1996, ApJ, 1996, 451, 821

Narayan, R., Barret, D., \& McClintock, J.E., ApJ, 1997, submitted

Rees, M.J. 1982 in The Galactic Center, AIP Conference Proceedings No 83, ed. G.R. Riegler \& R.D. Blanford (AIP: New York), 166

Smak, J. 1983, ApJ, 272, 234

Smak, J. 1993, Acta. Astron. 43, 101

van Paradijs, J. 1996, ApJ, 464, 139

Verbunt, F. 1996, in IAU Symp. 165, Compact Stars in Binaries, ed. E.P.J. van den Heuvel et al. (Dordrecht: Kluwer)

Warner, B., Livio, M., \& Tout, C. 1996, MNRAS in press

\section{Discussion}

M. Romanova: Some X-ray Novae show jets. You did not mention this fact in your talk. Are there any models which may explain jets? There are models where disc may be magnetically unstable and outflows are possible.

J.P. Lasota: I did not mention jets from SXTs because I don't like them. I am full of admiration for the observations of Felix Mirabel and Bob Hjellming, but I wish they didn't find jets. I have no explanation for them and I believe that what you call 'models' are no more than 'scenarios'. The possible connection between ADAFs and jets has been discussed by Ramesh Narayan at this workshop. 